\title{
Comparison of surgical outcomes between 3D and 2D VATS lobectomy for clinical stage I lung cancer
}

\section{Tae Ho Kim, Tae Hee Hong ${ }^{\star}$, Yong Soo Choi}

Department of Thoracic and Cardiovascular Surgery, Samsung Medical Center, Sungkyunkwan University School of Medicine, Seoul, Korea

Received: February 18, 2020

Revised: March 31, 2020

Accepted: April 9, 2020

Corresponding author:

Yong Soo Choi

Department of Thoracic and Cardiovascular Surgery, Samsung Medical Center, Sungkyunkwan University School of Medicine, 81 Irwonro, Gangnam-gu, Seoul 06351, Korea

Tel: +82-2-3410-6542

E-mail: choi.smcts@gmail.com

*Tae Ho Kim and Tae Hee Hong contributed equally to this study as first authors.

\begin{abstract}
Purpose: The use of three-dimensional (3D) thoracoscope provides improved depth perception and accuracy in video-assisted thoracoscopic surgery (VATS). This study aimed to compare the surgical outcomes between two-dimensional (2D) and 3D VATS lobectomy for stage I lung cancer.

Methods: From January 2013 to May 2018, 354 patients underwent VATS lobectomy and mediastinal lymph node dissection for clinical stage I lung cancer. The 3D VATS system was introduced in July 2016 in our center. A total of 182 patients underwent 2D VATS lobectomy from January 2013 to June 2016, and 172 patients underwent 3D VATS lobectomy from July 2016 to May 2018.

Results: The thoracotomy conversion rate was lower $(1.2 \%$ vs. $6.4 \%, P=0.011)$ in the $3 \mathrm{D}$ VATS group than in the 2D VATS group. Operation time (mean: 119.0 minutes vs. 151.4 minutes, $\mathrm{P}<0.001$ ), operative blood loss (median: $100 \mathrm{~mL}$ vs. $150 \mathrm{~mL}, \mathrm{P}<0.001$ ), and length of hospital stay after surgery (median: 5 days vs. 6 days, $P<0.001$ ) were significantly lower in the 3D VATS group than in the 2D VATS group. A higher number of lymph nodes was resected in 3D VATS (mean: 15.9 vs. $13.8, P=0.006$ ). No significant difference was observed between 3D and 2D VATS in terms of operative complications (Clavien-Dindo classification grade $\geq 1 ; 14.7 \%$ vs. $14.6 \%, P=0.322$ ). None of the patients died postoperatively.

Conclusion: Our retrospective data showed that the 3D thoracoscope had better surgical outcomes than 2D thoracoscope for VATS lobectomy in patients with clinical stage I lung cancer.
\end{abstract}

Keywords: Lung neoplasms; Thoracic surgery, video-assisted; Three dimensional
This is an Open Access article distributed under the terms of the Creative Commons Attribution Non-Commercial License (https:// creativecommons.org/licenses/ by-nc/4.0/).

\section{INTRODUCTION}

Thoracic surgery has traditionally been performed with open-thoracotomy incision and has 
rapidly developed into video-assisted thoracoscopic surgery (VATS) in the last 20 years. VATS is less invasive than the conventional thoracotomy approach and causes less trauma to the respiratory muscles. Therefore, VATS is less painful and has a quicker recovery time, and it reduces postoperative pulmonary function impairment and eventually reduces pulmonary complications [1,2]. Thus, two-dimensional (2D) VATS technology has been widely applied worldwide and has become a standard surgery for lung cancer.

However, the 2D image lacks depth, which may result in image distortion, impaired hand and eye coordination, and reduced ability to estimate size. As emerging imaging systems and techniques, three-dimensional (3D) VATS and robot-assisted thoracic surgery (RATS) have been developed. However, the RATS is extremely expensive and has limitations; thus, it is only used in large centers.

The 3D video systems are not as expensive as RATS and are easily applicable to small centers. It offers the advantage of improved depth perception and accuracy compared with 2D VATS [3]. The 3D video systems were first used in cholecystectomy and then expanded to other types of surgeries, which include colon resections, hernia repairs, hysterectomies, and urologic and prostate surgeries [4]. Since such systems have recently been applied in thoracic surgery and have been used for a short period of time, there is no consensus regarding its outcomes.

This study compared the safety of the surgery, postopera- tive complications, duration of surgery, and oncologic efficacy with 3D and traditional 2D VATS for stage I lung cancer.

\section{METHODS}

\section{Patient selection and study design}

The 3D VATS system was introduced in July 2016 in our center. We designed this study to compare the surgical outcomes of patients who underwent 3D and 2D VATS before the introduction of the 3D VATS technique. The records of all patients ( $n=377$ ) who underwent VATS lobectomy for stage I nonsmall cell lung cancer (NSCLC) by a single surgeon between January 2013 and May 2018 at the Samsung Medical Center were reviewed. We excluded 23 patients who underwent selective lymph node dissection, lymph node sampling, bilateral operation, or joint operation. In addition, 13 patients whose surgeries were converted to thoracotomy were not included. The reasons for such conversions were severe pleural adhesion, presence of anthraco-fibrotic lymph node, or extranodal invasion by a tumor (Supplementary Table 1).

After applying the exclusion criteria, 341 patients were enrolled in the study. Before the introduction of the 3D VATS technique, 2D VATS lobectomy was performed on $171 \mathrm{pa}$ tients between January 2013 and June 2016. A total of 170 3D VATS lobectomies were performed between July 2016 and May 2018. We compared the surgical outcomes of 3D VATS to those of 2D VATS (Fig. 1).

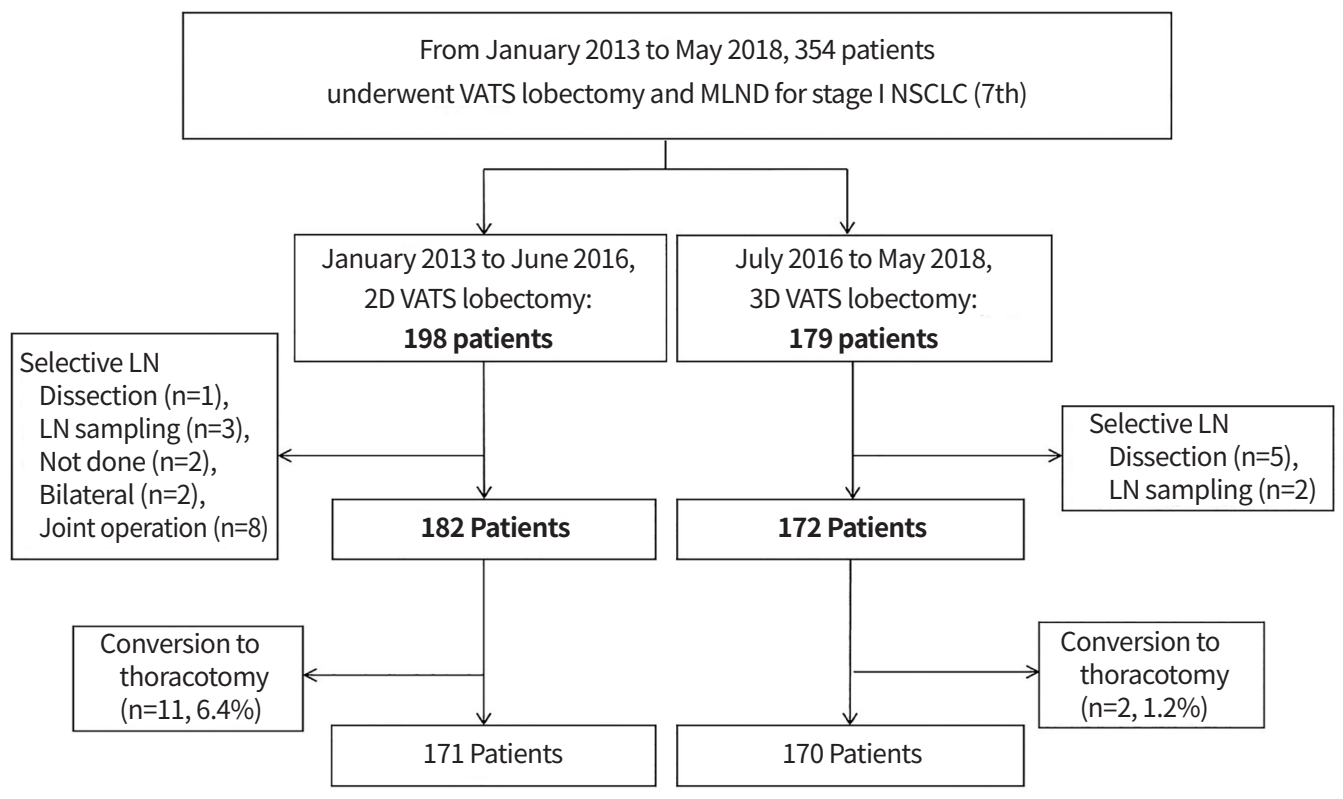

Fig. 1. Study populations. VATS, video-assisted thoracoscopic surgery; MLND, Mediastinal Lymph Node Dissection; NSCLC, non-small cell lung cancer; 2D, two-dimensional; 3D, three-dimensional; LN, lymph node. 


\section{PRECISION AND FUTURE MIEDICINE}

Tae Ho Kim, et al.

\section{Surgical technique}

All patients received general anesthesia with double-lumen endotracheal tube intubation and were placed in the lateral decubitus position, and single-lung ventilation was applied. The operator stood on the right side of the patient, and two assistants stood on the left side. The assistant rotated the camera $90^{\circ}$ toward the operator, providing the assistants on the left side the same view as during an open thoracotomy. The operator watched another monitor rotated $180^{\circ}$ to perform the surgery, with the same view as during open thoracotomy. In addition, similar surgical instruments were used for 3D and 2D VATS. Similar to when 3D surgery was started, the energy device (Thunderbeat, Olympus, Tokyo, Japan) was used more frequently.

The single-port VATS was always initiated with a mid-axillary 12-mm incision at the fifth intercostal space for pleural exploration. After an initial exploration of the pleural cavity through this incision with a $12-\mathrm{mm} 30^{\circ}$ thoracoscope, the $12-$ $\mathrm{mm}$ incision was extended to a 3.5 to $5-\mathrm{cm}$ incision at the mid-axillary line. Furthermore, 12-mm incisions at the mid-axillary line at the seventh intercostal spaces were added for the bi-port VATS lobectomy. In 3D VATS, we usually use the Karl Storz (KARL STORZ GmbH \& Co. KG, Tuttlingen, Germany) equipment consisting of $10-\mathrm{mm}, 30^{\circ}$ viewing angle $3 \mathrm{D}$ video thoracoscope with a 3D fiber optic cable.

In both groups, a wound protector (Endo Keeper, medium, Nelis, Bucheon, Korea) was applied, and anatomical resection of the veins, arteries, and bronchi was performed. Radical mediastinal lymph node dissection was routinely performed in the same manner in both groups. Once the operation was completed, a single chest tube was inserted into the anterior part of the incision for single port VATS and through the port at the seventh intercostal space for bi-port VATS. The details of the surgical techniques have been previously described by Chung et al. [5].

\section{Data acquisition}

Clinical information of all patients, including pulmonary function test results, operation time, volume of intraoperative bleeding, number of dissected lymph nodes and stations, the American Society of Anesthesiologists (ASA) Risk Scale score, hospitalization time after surgery, and complications, was collected. Intraoperative bleeding was visualized by an anesthesiologist in the suction bottle at the end of the operation. Data were based on the information from the medical records of the Samsung Medical Center. Histological type was established according to the World Health Organi- zation classification of lung cancer. TNM stage was determined according to the American Joint Committee on Cancer staging system, 7 th edition. The study proposal was approved by the ethics committee of Samsung Medical Center, which waived the need for informed consent (Institutional Review Board no. SMC 2020-01-080-001).

\section{Statistical analysis}

In the univariate analysis (age, sex, ASA class, clinical T stage, tumor location, pulmonary function test, histology, number of VATS port, complication rate, and re-operation rate), Fisher's exact test was used for comparisons between the $2 \mathrm{D}$ and 3 D VATS. The Mann-Whitney $U$ tests were used to evaluate data sets with continuous variables (operation time, estimated blood loss [EBL], and length of hospital stay). Continuous data were presented as the mean \pm standard deviation. Categorical variables were presented as frequency and percentages. Differences among the groups were examined using a two-way analysis of variance for continuous and categorical variables. A P $<0.05$ was considered statistically significant. Statistical analyses were performed with the SPSS software version 25 for Windows (IBM Co., Armonk, NY, USA).

\section{RESULTS}

\section{Clinical findings}

The prevalence of severe adhesion (diffuse and tight) was similar in 3D VATS group (11/182 [7.6\%]) and 2D VATS group $(13 / 172[6.0 \%])(P=0.318)$. The thoracotomy conversion rate was lower $(1.2 \%$ vs. $6.4 \%, P=0.011)$ in $3 D$ VATS than in $2 \mathrm{D}$ VATS. After applying the exclusion criteria, 171 patients remained in the 2D VATS group and 170 in the 3D VATS group. Diffusing capacity (DLCO) was significantly poorer (93.2\% vs. $89.1 \%, P=0.024)$ in 3D VATS than in 2D VATS. In terms of ASA risk scale score, the 3D VATS group had a higher score than the $2 D$ VATS group $(P=0.008)$. No other statistically significant differences were observed. The demographic characteristics of the patients are presented in Table 1.

\section{Pathological findings}

The total number of separately resected lymph nodes (13.8 vs. 15.9, $P=0.006)$ and the number of resected $N 2$ lymph nodes (8.6 vs. 9.9, $P=0.0 .035)$ significantly differed between the groups.

\section{Perioperative outcomes}

No in-hospital deaths or 90-day mortality was observed in ei- 
Table 1. Characteristics of patients undergoing VATS lobectomy and mediastinal lymph node dissection

\begin{tabular}{|c|c|c|c|}
\hline Characteristic & $\begin{array}{l}\text { 2D VATS } \\
(n=171)\end{array}$ & $\begin{array}{l}\text { 3D VATS } \\
(n=170)\end{array}$ & P-value \\
\hline Age (yr) & $61.7 \pm 8.7$ & $60.9 \pm 9.0$ & 0.396 \\
\hline Sex, male:female (female \%) & $82: 89(52.1)$ & $82: 88(51.8)$ & 0.959 \\
\hline ASA class & & & 0.008 \\
\hline 1 & $44(25.7)$ & $37(21.8)$ & \\
\hline 2 & $125(73.1)$ & $119(70.0)$ & \\
\hline 3 & $2(1.2)$ & $14(8.2)$ & \\
\hline Clinical T stage & & & 0.198 \\
\hline $\mathrm{T} 1 \mathrm{a}$ & $62(36.3)$ & $47(27.7)$ & \\
\hline $\mathrm{T} 1 \mathrm{~b}$ & $57(33.3)$ & $69(40.3)$ & \\
\hline $\mathrm{T} 2 \mathrm{a}$ & $52(30.4)$ & $54(31.8)$ & \\
\hline Tumor location & & & 0.270 \\
\hline LUL & $46(26.9)$ & $38(22.4)$ & \\
\hline LLL & $32(18.7)$ & $23(13.5)$ & \\
\hline RUL & $44(25.7)$ & $57(33.5)$ & \\
\hline RML & $15(8.8)$ & $11(6.5)$ & \\
\hline RLL & 34 (19.9) & $41(24.1)$ & \\
\hline \multicolumn{4}{|l|}{ Pulmonary function test } \\
\hline FEV1 & $94.5 \pm 14.0$ & $91.7 \pm 14.6$ & 0.072 \\
\hline FEV1/FVC & $75.4 \pm 8.1$ & $75.0 \pm 8.7$ & 0.682 \\
\hline DLCO & $93.2 \pm 16.1$ & $89.1 \pm 17.0$ & 0.024 \\
\hline Histology & & & 0.104 \\
\hline Adenocarcinoma & $150(87.7)$ & $149(87.6)$ & \\
\hline Squamous cell carcinoma & $14(8.2)$ & $13(7.7)$ & \\
\hline Etc. & $7(4.1)$ & $8(4.7)$ & \\
\hline
\end{tabular}

Values are presented as mean \pm standard deviation or number (\%). VATS, video-assisted thoracoscopic surgery; 2D, two-dimensional; 3D, three-dimensional; ASA, American Society of Anesthesiologists; LUL, left upper lobe; LLL, left lower lobe; RUL, right upper lobe; RML, right middle lobe; RLL, right lower lobe; FEV1, forced expiratory volume in 1 second; FVC, forced vital capacity; DLCO, diffusing capacity.

ther group. Operation time (mean: 119.0 minutes vs. 151.4 minutes, $\mathrm{P}<0.001$ ), operative blood loss (median: $100 \mathrm{~mL}$ vs. $150 \mathrm{~mL}, \mathrm{P}<0.001$ ), and length of hospital stay after surgery (median: 5 days vs. 6 days, $P<0.001$ ) were significantly lower in the 3D VATS group than in the 2D VATS. The operation time was further scrutinized to determine the confounding impact from the enhancement of the surgical skill (Supplementary Fig. 1). There was no significant difference of operation time within the early-and late-subgroups of 2D VATS and 3D VATS, whereas a significant reduction of operation time was confirmed after the introduction of 3D VATS $(P<0.001)$. No signif-
Table 2. Operative and postoperative outcomes of VATS lobectomy and mediastinal node dissection

\begin{tabular}{lccc}
\hline Variable & $\begin{array}{c}\text { 2D VATS } \\
(\mathrm{n}=171)\end{array}$ & $\begin{array}{c}\text { 3D VATS } \\
(\mathrm{n}=170)\end{array}$ & P-value \\
\hline VATS port & & & $<0.001$ \\
$\quad$ Single & $43(25.1)$ & $18(10.6)$ & \\
$\mathrm{Bi}$ & $53(31.2)$ & $151(88.8)$ & \\
Tri & $75(43.9)$ & $1(0.6)$ & \\
Operation time (min) & $151.4 \pm 40.8$ & $119.0 \pm 35.3$ & $<0.001$ \\
Estimated blood loss (mL) & $150(100-200)$ & $100(50-113)$ & $<0.001$ \\
Lymph node dissection & & & \\
Total resected (separately) & $13.8 \pm 7.1$ & $15.9 \pm 7.2$ & 0.006 \\
N2 resected & $8.6 \pm 5.7$ & $9.9 \pm 5.6$ & 0.035 \\
Examined N2 station & $2.8 \pm 0.8$ & $3.0 \pm 0.8$ & 0.025 \\
Hospital stay (day) & $6(4-8)$ & $5(4-7)$ & $<0.001$ \\
Complication (grade $\geq 1)$ & $25(14.6)$ & $25(14.7)$ & 0.982 \\
Re-operation & $1(0.6)$ & $2(1.2)$ & 0.623 \\
In-hospital death & 0 & 0 & 1.000 \\
& & &
\end{tabular}

Values are presented as number (\%), mean \pm standard deviation, or median (range).

VATS, video-assisted thoracoscopic surgery; 2D, two-dimensional; 3D, three-dimensional.

icant difference was observed between 3D and 2D VATS in terms of operative complications (Clavien-Dindo classification Grade $\geq 1 ; 14.7 \%$ vs. $14.6 \%, P=0.322$ ) (Table 2 ).

\section{DISCUSSION}

In this retrospective study of 341 patients with clinical stage I NSCLC, the 3D thoracoscope provided better surgical outcomes than 2D thoracoscope for VATS lobectomy. Based on the result of the surgery in the two groups with similar baseline clinical characteristics using different video systems, the 3D VATS group had a higher number of resected lymph nodes (Supplementary Table 2), lower operative blood loss, reduced operative time, and quicker recovery time than the $2 \mathrm{D}$ VATS group. No significant differences were observed between the two groups in terms of complications and acute phase postoperative outcomes.

In actual clinical practice, the extent of lymph node dissection in early-stage NSCLC cases may be different from that of advanced stage cases. Metastasis of tumor cells through the pathway of lymph drainage to the regional lymph nodes is rare during the early stage [6]. Considering the results of the lobe-specific patterns of nodal metastases, the concept of 
selective lymph node dissection was proposed [7-9].

Several reports have shown that selective lymph node dissection or lymph node sampling at an early stage is favorable for disease-free survival and overall survival compared with complete lymph node dissection [10-14]. Because the number of complications may increase when aggressive and extensive dissection of the lymph nodes is performed in patients with early-stage lung cancer, such as pure ground glass opacity, there is a concern regarding whether systematic complete lymph node dissection should be performed if the adhesion is severe and node dissection is challenging to perform. The proposed optimal number of lymph nodes to be dissected to prevent staging errors have been shown in other studies $[9,15]$. In this study, the number of separately resected lymph nodes was 13.8 in 2D VATS and 15.9 in 3D VATS, and the optimal number of lymph nodes dissected was similar to that of other studies.

Results of previous comparisons of 3D and 2D VATS showed no difference in terms of postoperative complications, EBL, and number of harvested lymph nodes $[16,17]$. The only significant difference in most studies was in terms of the reduced operation time. The results of this study were slightly different.

In comparison of the patient groups, most conditions are similar, but 3D VATS group was poorer because DLCO is statistically significantly lower. The surgical procedures were similar. However, the number of ports was slightly different depending on the surgical time. 2D VATS was performed with single and tri-port (69\%), but most 3D VATS were performed with bi-port (88.8\%) and single and tri-ports (11.2\%). The total number of separately resected lymph nodes was 13.8 in 2D VATS and 15.9 in 3D VATS. In addition, the number of resected N2 and examined N2 stations significantly increased. Using the 3D video system, lymph node dissection was less challenging to perform, and a more extensive dissection can be carried out in a shorter period of time.

Unlike in other reports, EBL also decreased ( $150 \mathrm{~mL}$ vs. 100 $\mathrm{mL}$ ). This could be attributed to the effect of an energy device (Thunderbeat) used at the same time as the 3D VATS.

Subgroup analysis was performed to reduce errors due to differences in the number of ports. The demographic characteristics of the patients are presented in Supplementary Table 3. No statistically significant differences were observed between two groups. The surgical outcome of the subgroup analysis still showed a significant difference in operation time, EBL and hospital stay. However, the advantage in a resected number of lymph node dissections identified in the entire 3D VATS group disappeared (Supplementary Table 4).
Despite these advantages, patients who underwent 3D VATS initially presented with headache and ocular fatigue [18]. These problems have recently improved with the use of 3D glasses. 3D imaging improves depth perception, spatial location, and precision of the surgical performance particularly during advanced technical maneuvers [19].

In conclusion, our retrospective data showed that the 3D thoracoscope had better surgical outcomes than 2D thoracoscope for the treatment of early-stage lung cancer. Further studies comparing the benefits and indications of 3D VATS lobectomy must be conducted.

\section{CONFLICTS OF INTEREST}

No potential conflict of interest relevant to this article was reported.

\section{ORCID}

Tae Ho Kim https://orcid.org/0000-0002-2084-6391

Tae Hee Hong https://orcid.org/0000-0003-2708-8893

Yong Soo Choi https://orcid.org/0000-0001-8492-7644

\section{AUTHOR CONTRIBUTIONS}

Conception or design: THK, YSC.

Acquisition, analysis, or interpretation of data: THK, THH, YSC.

Drafting the work or revising: THK, THH, YSC.

Final approval of the manuscript: THK, THH, YSC.

\section{REFERENCES}

1. Onaitis MW, Petersen RP, Balderson SS, Toloza E, Burfeind WR, Harpole DH Jr, et al. Thoracoscopic lobectomy is a safe and versatile procedure: experience with 500 consecutive patients. Ann Surg 2006;244:420-5.

2. Scott WJ, Howington J, Feigenberg S, Movsas B, Pisters K; American College of Chest Physicians. Treatment of nonsmall cell lung cancer stage I and stage II: ACCP evidencebased clinical practice guidelines (2nd edition). Chest 2007; 132(3 Suppl):234S-42S.

3. Yamauchi Y, Shinohara K. Effect of binocular stereopsis on surgical manipulation performance and fatigue when using a stereoscopic endoscope. Stud Health Technol Inform 2005;111:611-4.

4. McLachlan G. From 2D to 3D: the future of surgery? Lancet 
2011;378:1368.

5. Chung JH, Choi YS, Cho JH, Kim HK, Kim J, Zo Jl, et al. Uniportal video-assisted thoracoscopic lobectomy: an alternative to conventional thoracoscopic lobectomy in lung cancer surgery? Interact Cardiovasc Thorac Surg 2015;20:813-9.

6. Kim AW. Lymph node drainage patterns and micrometastasis in lung cancer. Semin Thorac Cardiovasc Surg 2009; 21:298-308.

7. Cerfolio RJ, Bryant AS. Distribution and likelihood of lymph node metastasis based on the lobar location of nonsmallcell lung cancer. Ann Thorac Surg 2006;81:1969-73.

8. Rami-Porta R, Wittekind C, Goldstraw P; International Association for the Study of Lung Cancer (IASLC) Staging Committee. Complete resection in lung cancer surgery: proposed definition. Lung Cancer 2005;49:25-33.

9. Okada M, Sakamoto T, Yuki T, Mimura T, Miyoshi K, Tsubota N. Selective mediastinal lymphadenectomy for clinico-surgical stage I non-small cell lung cancer. Ann Thorac Surg 2006;81:1028-32.

10. Watanabe S, Asamura H, Suzuki K, Tsuchiya R. The new strategy of selective nodal dissection for lung cancer based on segment-specific patterns of nodal spread. Interact Cardiovasc Thorac Surg 2005;4:106-9.

11. Darling GE, Allen MS, Decker PA, Ballman K, Malthaner RA, Inculet RI, et al. Randomized trial of mediastinal lymph node sampling versus complete lymphadenectomy during pulmonary resection in the patient with $\mathrm{NO}$ or N1 (less than hilar) non-small cell carcinoma: results of the American College of Surgery Oncology Group Z0030 Trial. J Thorac Cardiovasc Surg 2011;141:662-70.

12. Huang X, Wang J, Chen Q, Jiang J. Mediastinal lymph node dissection versus mediastinal lymph node sampling for early stage non-small cell lung cancer: a systematic review and meta-analysis. PLoS One 2014;9:e109979.

13. Adachi H, Sakamaki K, Nishii T, Yamamoto T, Nagashima T, Ishikawa Y, et al. Lobe-specific lymph node dissection as a standard procedure in surgery for non-small cell lung cancer: a propensity score matching study. J Thorac Oncol 2017;12:85-93.

14. Gajra A, Newman N, Gamble GP, Kohman LJ, Graziano SL. Effect of number of lymph nodes sampled on outcome in patients with stage I non-small-cell lung cancer. J Clin Oncol 2003;21:1029-34.

15. Ludwig MS, Goodman M, Miller DL, Johnstone PA. Postoperative survival and the number of lymph nodes sampled during resection of node-negative non-small cell lung cancer. Chest 2005;128:1545-50.

16. Dong S, Yang XN, Zhong WZ, Nie Q, Liao RQ, Lin JT, et al. Comparison of three-dimensional and two-dimensional visualization in video-assisted thoracoscopic lobectomy. Thorac Cancer 2016;7:530-4.

17. Bagan P, De Dominicis F, Hernigou J, Dakhil B, Zaimi R, Pricopi $\mathrm{C}$, et al. Complete thoracoscopic lobectomy for cancer: comparative study of three-dimensional high-definition with two-dimensional high-definition video systems. Interact Cardiovasc Thorac Surg 2015;20:820-3.

18. Hanna GB, Shimi SM, Cuschieri A. Randomised study of influence of two-dimensional versus three-dimensional imaging on performance of laparoscopic cholecystectomy. Lancet 1998;351:248-51.

19. Lusch A, Bucur PL, Menhadji AD, Okhunov Z, Liss MA, Perez-Lanzac A, et al. Evaluation of the impact of three-dimensional vision on laparoscopic performance. J Endourol 2014;28:261-6. 